\title{
Chasing Play on TikTok from Populations with Disabilities to Inspire Playful and Inclusive Technology Design
}

\author{
Jared Duval \\ University of California Santa Cruz \\ Santa Cruz, California \\ Jduval@ucsc.edu \\ Melissa Chu* \\ University of California Santa Cruz \\ Santa Cruz, California \\ Geoffrey Xiang* \\ University of California Santa Cruz \\ Santa Cruz, California
}

\author{
Ferran Altarriba Bertran \\ University of California Santa Cruz \\ Santa Cruz, California \\ faltarri@ucsc.edu
}

Divya Subramonian*

University of California Santa Cruz

Santa Cruz, California

Sri Kurniawan

University of California Santa Cruz

Santa Cruz, California

skurnia@ucsc.edu

\author{
Siying Chen ${ }^{*}$ \\ University of California Santa Cruz \\ Santa Cruz, California
}

\author{
Austin Wang* \\ University of California Santa Cruz \\ Santa Cruz, California \\ Katherine Isbister \\ University of California Santa Cruz \\ Santa Cruz, California \\ katherine.isbister@ucsc.edu
}

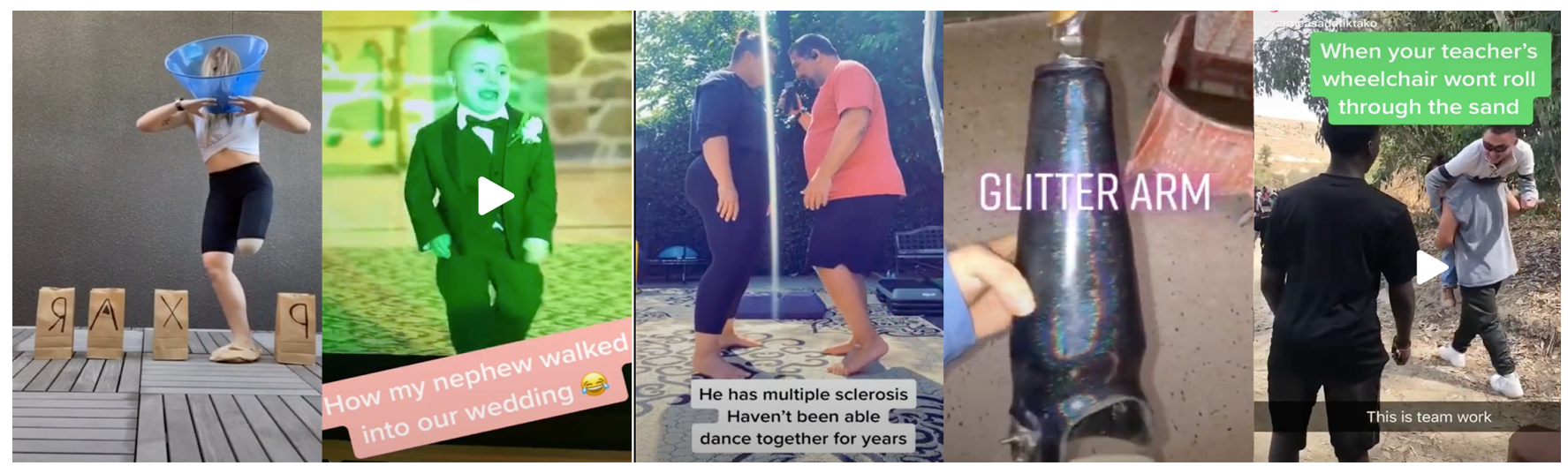

Figure 1: Screenshots of TikTok videos that can can inspire playful technology design inclusive of people with special needs.

\section{ABSTRACT}

There is an open call for technology to be more playful $[5,79]$ and for tech design to be more inclusive of people with disabilities [80]. In the era of COVID19, it is often unsafe for the public in general and people with disabilities, in particular, to engage in inperson design exercises using traditional methods. This presents a missed opportunity as these populations are already sharing playful content rich with tacit design knowledge that can be used to inspire the design of playful everyday technology. We present our process of scraping play potentials [4] from TikTok from content creators with disabilities to generate design concepts that may inspire future technology design. We share 7 emerging themes from the scraped

${ }^{*}$ High school students affiliated with UCSC through Summer Internship Program

This work is licensed under a Creative Commons Attribution International 4.0 License.

CHI '21, May 8-13, 2021, Yokohama, Japan

(C) 2021 Copyright held by the owner/author(s).

ACM ISBN 978-1-4503-8096-6/21/05.

https://doi.org/10.1145/3411764.3445303 content, a catalog of design concepts that may inspire designers, and discuss the relevance of the emerging themes and possible implications for the design concepts.

\section{CCS CONCEPTS}

- Human-centered computing $\rightarrow H C I$ design and evaluation methods; User centered design; Contextual design; Accessibility design and evaluation methods;

\section{KEYWORDS}

Chasing Play, Populations with Special Needs, Play Potentials, Inclusive Design, Social Media, TikTok, Design Concepts, Disability

\section{ACM Reference Format:}

Jared Duval, Ferran Altarriba Bertran, Siying Chen, Melissa Chu, Divya Subramonian, Austin Wang, Geoffrey Xiang, Sri Kurniawan, and Katherine Isbister. 2021. Chasing Play on TikTok from Populations with Disabilities to Inspire Playful and Inclusive Technology Design. In CHI Conference on Human Factors in Computing Systems (CHI '21), May 8-13, 2021, Yokohama, Japan. ACM, New York, NY, USA, 15 pages. https://doi.org/10.1145/3411764. 3445303 


\section{INTRODUCTION}

Two mainstream ways that technology can be designed to accommodate users with disabilities are by creating technology that provides a specific service to people with disabilities (Assistive Technology) or making general technology more accessible to people with disabilities (Accessible Technology) [89]. It is very pragmatic and vital to include people with disabilities in the design process in both cases. This paper is motivated by the need to include populations with disabilities to inspire design more broadly and generally, looking at values other than efficiency and accessibility. We design better technology for everyone when we include people with disabilities early and often in the process [80].

We are particularly interested in designing technology that supports play. Play for its own sake is valuable [17]. Play and playfulness can have a positive impact on the well-being of individuals and groups $[19,44]$. Play provides us with the agency to be creative, express ourselves, and learn [75, 78]. Play creates opportunities for meaningful social connection [45]. Play is universal to all humans (and possibly all living creatures) [17], including humans with disabilities. People with disabilities are playful (e.g. [39]) and how they play [41] can benefit the design of technology and improve society at large. Playful technology designed with people with disabilities has the potential to educate the general population about people with disabilities, increase the visibility of people with disabilities, and support social relationships between people regardless of their disability status.

It can be challenging to include people with disabilities for participatory work generally [85], but there is a specific added risk during a global pandemic. In addition to general guidelines that limit in-person contact, populations of people with disabilities often have medical needs that place them at higher risk from the COVID19 virus [7]. In light of quarantine, designers are employing creative methodologies to carry out remote design work that is usually done in situ [87] (e.g., using games to educate the public about COVID19 and collect data [53]). Many of these technologies are not accessible to people with disabilities [6]. Co-Design sessions should be valuable to all parties [18], but they disrupt everyday life and require participants to invest their precious time. We are interested in tapping into the tacit and contextual design knowledge of people with disabilities on platforms they are already using to share content for all of these reasons. Social media is rife with potential design material. We chose to focus on TikTok because is an inherently playful social media platform and it is a safe way to engage with populations of people with disabilities during a pandemic in a way that is not disruptive to their everyday life. To capture play potentials [3] on TikTok we employed the Situated Play Design methodology [4]. These play potentials inspired design concepts that could inspire future technology.

In this paper, we present a catalog of design concepts to inspire future playful technology based on play potentials from people with disabilities. To arrive at our design concepts, we chased play potentials on TikTok by scraping videos from content creators with disabilities. We analyzed the scraped content and present 7 emerging themes that helped inspire and generate the design concepts. Finally, we discuss the relevance of the 7 emerging themes and our design concepts' possible implications.

\section{BACKGROUND}

In technology design, people with disabilities are usually included to make general technology more accessible or make a technology solution to address a specific need for a population of people with disabilities, which is discussed in the first section. Next, we discuss some examples of playful technologies designed with and for people with disabilities to achieve utilitarian goals. We then discuss standard methods of how these populations are usually included in the design process, but how play is not usually the goal, whereas play is our work's goal. Finally, we discuss how Situated Play Design (SPD) [4] is an appropriate methodology to design technology inspired by how people with disabilities play.

\subsection{Accessible and Assistive Technology}

Accessible technology can be used by all people in the target audience regardless of disability status [80]. Technology that is not accessible places a handicap [9] on people who do not have equitable access to the services that technology is designed to provide [38]. A handicap is not a person's disability-it is the barriers that society and technology place on people with disabilities [38]. Too often, making technology accessible is an act of retrofitting solutions to make the original tech usable by people with disabilities [61], when these populations should have been included in the first place, resulting in designs that are more accessible and userfriendly for everyone [80]. Assistive technology is meant to serve a specific need of a population of people with disabilities and is meant to be used primarily by people with disabilities, caretakers, and medical professionals [76]. Assistive technology should be accessible to the target population so that they can use it-and common co-occurring disabilities should also be considered [83]. Technology design that includes people with disabilities often naturally becomes playful or includes elements of play, which results in novel and interesting user experiences (e.g. [30, 31, 35, 82]). In these cases, play emerged naturally, but may not have been sought out directly. We are interested in designing technology that is directly inspired by play.

\subsection{Playful Technologies and People with Disabilities}

As is true for people in general, people with disabilities are playful [39]. Play and games can serve people with disabilities in numerous ways, including increasing their visibility, improving public perception of people with disabilities, and fostering healthy connections in communities. Public visibility of people with disabilities and designing for social acceptance can reduce stigmas [74]. Negative socio-cultural stigma continues to dissuade people from using their assistive technology [67], isolate themselves, and worry about unwanted attention [24, 47, 49, 64]. Oppression of people with disabilities is systematic, political, and sociocultural [21]. Historically, people with disabilities have put in the labor to improve their rights (e.g., the disability rights movement [21]), but all of society should actively participate in the shared responsibility. Play has the potential to make some of this labor feel less like work. In this work, we aim to create playful design concepts inspired by people with disabilities and speculate [8] a future where these technologies 
can playfully support societal growth and opportunities for the inclusion of people with disabilities.

2.2.1 Play. Play is an apt vehicle to bring about societal change and improve the quality of life of people with disabilities because it embodies the very qualities of growth and pleasure: it is biologically evolved to help us learn and build skills [17], it is naturally social [73, p. 9-10], and the voluntary nature of play allows us to focus on the style of play over the outcome [12]. Play captivates us all: scientists, researchers, parents, children, teachers, and adults. It can be thought of as a biological trait [17], an attitude [72], a phenomenological state [48], a set of properties [17], a state of mind free from time [22], a process [28], or an educational tool [14]. Even purposeless play-or paratelic play-is useful because it provides an opportunity for breaks, which are necessary components of serious activities like work and dismantling oppression [81]. Play can elicit a sense of pronoia or the suspicion that the universe is conspiring to help [55]. Play provides a continuation desire. "Play trains our physical skills, sharpens our mental abilities, and deepens our insights into our social capabilities" [28]. Play helps everyone.

Play can be employed for pragmatic purposes, including helping people with disabilities. Telic play is a serious mindset where we engage in play for a specific purpose, while paratelic play is a playful state where play itself is the goal [79]. When play is telic, it often becomes constrained, contextualized, and structured to achieve a premeditated purpose. One example is play therapy [16] Play therapy has been effective for children with schizophrenia, enuresis/encopresis, anxiety disorders, trichotillomania, selective mutism, withdrawn behavior, acting out behaviors, sexual abuse, trauma and neglect, learning/academic problems, and various life adjustment problems $[15,16]$. It has been used with adults for couples therapy, stress management, group therapy for older adults, and psychotherapy [71]. Play carries meanings with significant resonance for older adults [43].

2.2.2 Games. Structured play inherently leads to thinking about games [90]. Playfulness does not need to have the formal structure of games [54]. Games are generally beneficial for the same reasons play is generally beneficial. They improve our cognitive and social skills [20,37]. Games are used to teach us about politics [58], experiences of others [23], and education [68]. Games can make us superheroes of our own life and health [56]. Serious games for health [86] have the potential to offer people with disabilities a cost-effective, personalized, data-driven, connected, and motivating context for otherwise tedious and repetitive therapy. Their underlying systems are designed to give us a reason to keep trying [33] A variety of serious games for health had been documented to be effective for their target populations, and they are wide-ranging in their platforms, health outcomes, and target populations, from an exergame to help blind children with balance [60] to embodied persuasive games for adults in wheelchair [36], to speech therapy for children with cleft [27], to mobile games for motivating tobaccofree life in early adolescence [65]. Video games improved 69\% of psychological therapy outcomes, $59 \%$ of physical therapy outcomes, $50 \%$ of physical activity outcomes, $46 \%$ of clinician skills outcomes, $42 \%$ of health education outcomes, $42 \%$ of pain distraction outcomes, and $37 \%$ of disease self-management outcomes [66].

\subsection{Designing Assistive and Accessible Technology}

By their nature, several design methods are potentially supportive of the participation of people with disabilities for creating assistive and accessible technology [84]. Participatory Design [62] encompasses a variety of methodologies that can productively and effectively include people with disabilities as co-designers including focus groups [32], wizard of oz [25], cultural probes [34], brainstorming [88], and bodystorming [63] to name a few. To successfully implement these design methods, it helps to take an ability-focused approach [89]. When people with disabilities are included early and often in the design process, the resulting design artifact is often universally accessible and more usable by everyone [80]. In both participatory design and universal design, the inclusion of people with disabilities is not meant to benefit only a minority of the population, but rather everyone who engages with the design [80]. Playful technology inspired by people with disabilities has the potential to be universally beneficial for anyone interested in engaging. Many of our design concepts, described later, are potentially exciting to people regardless of their disability status.

\section{RESEARCH METHOD}

Situated Play Design (SPD) [5] is an apt methodology for our work because it allows us to unearth playful behaviors from people with disabilities so that we can share them with fellow designers to inspire future technology towards contributing to future designs, either those to support play wholly or those with other purposes where incorporating playful aspects could be beneficial. Situated Play Design is an extension to existing play design approaches that focuses on uncovering existing manifestations of contextual play as a starting point for designing for situated and emergent playful engagement [3]. The playful interactions that exist and emerge naturally as people with disabilities engage in their everyday context and activities are likely meaningful to them [5]. SPD supports designers in uncovering contextual play manifestations, called play potentials, and using them as foundations of design intervention, following three main steps: First, designers chase naturally existing or spontaneously emerging forms of play when interacting with users in (semi-) naturalistic settings. Second, a design intervention is created to support and enhance those forms of play. Third, this design intervention is deployed in the wild, where its impact can be evaluated. These steps can be iterated until a satisfactory design is achieved [5]. Due to the safety concerns related to COVID19 and the higher health risk status of many people with disabilities, we employed a contact-free approach to the SPD methodology. SPD is an open methodological frame flexible enough to complete all three steps of chasing play potentials during the constraints of conducting research during the COVID19 pandemic. For the first step, we chased play potentials and their contextual manifestations by scraping existing content from content creators on TikTok with disabilities using a Netnography-style approach [51], which we describe in depth in Scraping Content below. For the second step, we took a generative approach to develop a catalog of design concepts that could inspire future playful technology artifacts inspired by the play potentials we scraped, described in depth in Designing Concepts below. For step 3, we formed intermediate-level knowledge 
via our 7 emerging themes and we envisioned the impact of our designs in the Discussion by imagining speculative futures [8]. This work provides an opportunity for "design after design" [13] when we are not constrained by current technical limitations and after the pandemic when it is safer to engage with populations with disabilities. The specific research questions that drove this work are:

RQ1 What play potentials exist from scraping content on TikTok from creators with disabilities?

RQ2 What themes emerge from an analysis of scraped play potentials, and how does the TikTok platform influence the results?

RQ3 What kinds of designs can the scraped play potentials inspire, and how might these designs affect society?

Due to the COVID19 pandemic, we wanted to safely and nonintrusively chase play potentials from these populations as part of our broader research agenda [5], which is why we chose to scrape existing content on social media. We chose TikTok because: (1) it hosts massive amounts of playful content, (2) the video format affords capturing interactions and often more context than images, (3) people with disabilities have already adopted the platform, and (4) features of the app allow us to find relevant content, such as the keyword search and the "For You" page described below.

We mentored 5 high school students (who are authors of this paper) over an 8-week summer internship through a program called Science Internship Program who worked on various computational media projects related to designing and creating technology for people with disabilities, including this project. The 5 high school interns did the majority of the content scraping, data analysis, and design concept sketching, under our guidance. All high school interns completed the required training for conducting ethical research on human subjects, received the training certificate, and are officially in our research protocol approved by our institutional review board. All high school interns watched recorded lectures from undergraduate classes that researchers taught to conduct a qualitative/thematic analysis. All interns worked full-time with researchers and were highly supervised, including daily meetings and regular auditing of their work. All interns were exceptionally brilliant high performing students in the top $10 \%$ of their high schools-and all were members of historically disadvantaged communities and minorities. Classically trained HCI Researchers were responsible for overseeing the high school interns, aggregating the coded data, computing the agreement, and presenting the results.

\subsection{Scraping Content}

TikTok logs videos that users interact with and the creators that people follow to train an algorithm that curates custom suggestions hosted on the main page, the "For You" page. Each student created a new TikTok account so that the curation algorithm would start with a clean slate. For the first week, the interns spent an hour a day liking videos that they thought featured an interaction or behavior that was susceptible to leading to a play potential (liberally defined). By doing that, they trained the algorithm to highlight playful content on the "For You" page. For the second week, the students started to train the algorithm to curate playful content from creators with disabilities by following creators with disabilities and favoriting playful videos featuring people with disabilities.
To seed the algorithm, the highschoolers searched for relevant hashtags such as \#disability, \#disabled, \#blind, \#deaf, \#signLanguage, \#cerebralPalsy, \#accessibility, \#colorBlind, \#impairment, \#handicap, \#autism, \#ASD, \#play, \#game, \#fun, and \#haha. The keywords included general terms related to disability, specific disabilities, and terms to influence the algorithm to show playful content. Videos that contained play potentials were logged into a Google Sheet (provided in the supplementary materials) that contains a link to the video, the content creator's handle, a brief description of the video, and space for the students to list keywords and themes (RQ1). To ensure the integrity of access to the content for data analysis, we also stored all logged videos and a link to the copy, which was prudent because of the potential bans to TikTok that took place after we started. We asked interns to make quick 10-minute daily diary entries to reflect on the experience and process of scraping content on TikTok from users with disabilities and doing design work as an autoethnographic exercise [29]. By the third week, the "For You" page was populated with relevant videos from people with disabilities containing play potentials. When the content was stale, the interns seeded the algorithm with more relevant hashtags and logged relevant videos from their searches. The research team met weekly to update the hashtags bank and discuss tactics for seeding the algorithm to continue curating relevant content. The interns scraped content for an hour a day, 5 days a week, for 4 weeks (inclusive of the time it takes to log videos in the spreadsheet).

\subsection{Data Analysis}

Students independently generated a list of themes for the videos and then collaborated as a group to propose a set of combined themes to the research mentors (RQ2). Research mentors created the final theme names to be concise and descriptive during a group meeting. The 7 themes are non-exclusive and are discussed in detail in the Emerging Themes section. Interns spent an hour a day for a week analyzing the logs and tagging relevant themes. We hypothesized that TikTok is a very performative platform with a few introspective reflection instances. To better understand how the platform impacted our results (RQ2), interns placed each play potential on a spectrum of introspective to performative. Interns highlighted exemplars (their favorite play potentials) and listed technology mediums that might be relevant such as mobile devices, the internet of things, and wearables. The completed analysis is included in the supplementary materials. We highlighted exemplars because we wanted to design technology directly inspired by exciting play potentials in context. We added possibly relevant technology mediums to inspire ourselves to think about diverse application possibilities.

\subsection{Designing Concepts}

For week six of the internship, we asked interns to brainstorm 1 concept individually for each theme. We had them work individually to assess each student's individual strengths. In weeks 7 , students worked in pairs to brainstorm more ideas and present them to the larger group. Without any prior design experience, we challenged students to ignore the current limitations of technology, to design around playful moments instead of entire systems, to be specific, to present their ideas visually, and to think beyond 
"helping" people with disabilities. We gave these directions because speculative design [8] plays creatively with current technology limitations to imagine futuristic-yet-plausible designs in ways that transgress existing design directions. Mentors then facilitated a group brainstorming session where we watched TikTok videos marked as exemplars selected by students and then let students pick resulting design ideas to create sketches. In week 8 , mentors provided feedback and tools on improving their design concept sketches used in the interns' final presentation for the program. The mentors then did one final iteration on the interns' designs presented in the Design Concept Catalog section of this paper (RQ3). We reflect on these design concepts' possible implications in the "Discussion" section of this paper (RQ3).

\subsection{Ethics}

The high school interns participating in the Science Internship Program at University of California Santa Cruz are minors. Interns' parents or legal guardians signed consent forms to allow them to participate in summer research projects, including the work presented here. In addition, all high school summer interns completed the required training for conducting ethical research on human subjects provided by University of California Santa Cruz, received the training certificate, and are officially listed in our research protocol approved by our institutional review board.

This research was reviewed and approved by our institutional ethics review board. A tricky element of our research is scraping existing content on social media and the ethical implications of researching on these platforms. Our data collection method closely aligns with Netnography[51], which has established ethical guidelines [50]. These include the notion of public versus private information on social media, whether to anonymize or cite participants, and informed consent. Kozinets argues that ethical procedures must be decided on a case-by-case basis contingent upon the topic matter, the research purposes and the research approach of the particular netnography [51]. Some platforms such as Facebook and Instagram have varying levels of security and privacy settings for content and profiles that complicate what is truly public. Researchers using platforms with privacy settings must respect what is considered public and not. TikTok is designed to be entirely public-facing. TikTok is not designed to group people, create small social circles, or share private information to subsets of people-instead, it is an open broadcasting platform where content creators try to reach as many people as possible, strangers or otherwise. Bassett and Kozinets argue that when the internet is used as a "megaphone-like" public broadcasting medium [57], we can thus perceive it as a form of cultural production, in a similar framework to that of the print media, broadcast television and radio where we should cite the source so that broadcasters can be credited for their work $[11,50]$. Because this research is minimal risk and fits the notion of public broadcasting online, we provided links to the original content in our published materials to respect the creators' (ongoing) decisions concerning public access to the videos. Netnography of public archival content (not active research interventions such as interviewing) like the scraping methodology employed in this research would be unduly complicated with informed consent because the manual, non-automated access by researchers of public information should be acceptable without special permissions or actions [1] and removing information from unreachable broadcasters would undermine researchers' ability to contribute to society [50]. Therefore, we included all applicable data scraped in our supplementary materials and make every effort to represent the content in this publication respectfully and in a positive light. Finally, our data was manually scraped without any automated system or software and is not used for commercial purposes, and therefore, at the time of writing, adheres to TikTok's terms of services.

\section{EMERGING THEMES}

5 high school students individually analyzed their subset of videos to categorize them into the 7 emerging themes. Each theme is discussed in detail in the following sections. There were 285 unique videos total and 24 videos analyzed by two or more students, causing an overlap (RQ1). The theme categories are nominal, non-mutually exclusive, and there were 5 total raters. Between all 7 themes in the 24 overlapping videos, two or more raters agreed on whether or not a video belonged to a theme 147 times and disagreed 27 times, resulting in an average agreement of $84.48 \%$. While scraping content, interns marked exemplar videos that showcased play potentials they felt could directly inspire technology design. The number of videos in each theme and the number of exemplars marked for each theme is shown in Table 1.

Table 1: Distribution of TikTok videos by theme

\begin{tabular}{lcc}
\hline Theme & Total & Exemplars \\
\hline Everyday Theatrical Life Sketches & 130 & 11 \\
Playful Advocacy & 110 & 7 \\
Debunking Myths/Stereotypes & 24 & 7 \\
Gamification Therapy/Rehab & 51 & 25 \\
Impossible Challenges & 12 & 7 \\
Perks of my Disability & 10 & 1 \\
Duet Differences & 12 & 3 \\
\hline
\end{tabular}

Some people with disabilities use TikTok to log their personal reflections, while some use it to reach an audience-the distinction is whether the video was originally recorded for oneself or others. We hypothesized that the performative nature of TikTok and the completely public-facing broadcast affordances of the app would lead to videos that trend outwards from self (away from introspective and reflective content). Therefore, raters placed each video on a 5 point Likert scale where 1 is totally introspective (like a diary entry), 3 is elements of both, and 5 is totally performative (like a talent show). The resulting mean is 3.7 with a standard deviation of 1.38 , and a skew of -0.72 , confirming our hypothesis that content on TikTok is generally more outward-facing. As shown in the top chart of Figure 2, the frequency of the rating is highly skewed towards performative, where points were averaged and rounded when multiple raters analyzed the same video. These results conclude that the TikTok platform does impact the type of play potentials that can be scraped from the platform (RQ2) and, therefore, the types of designs we can expect it to inspire. Interns were also asked to imagine what technology medium might be most interesting to design for, given 


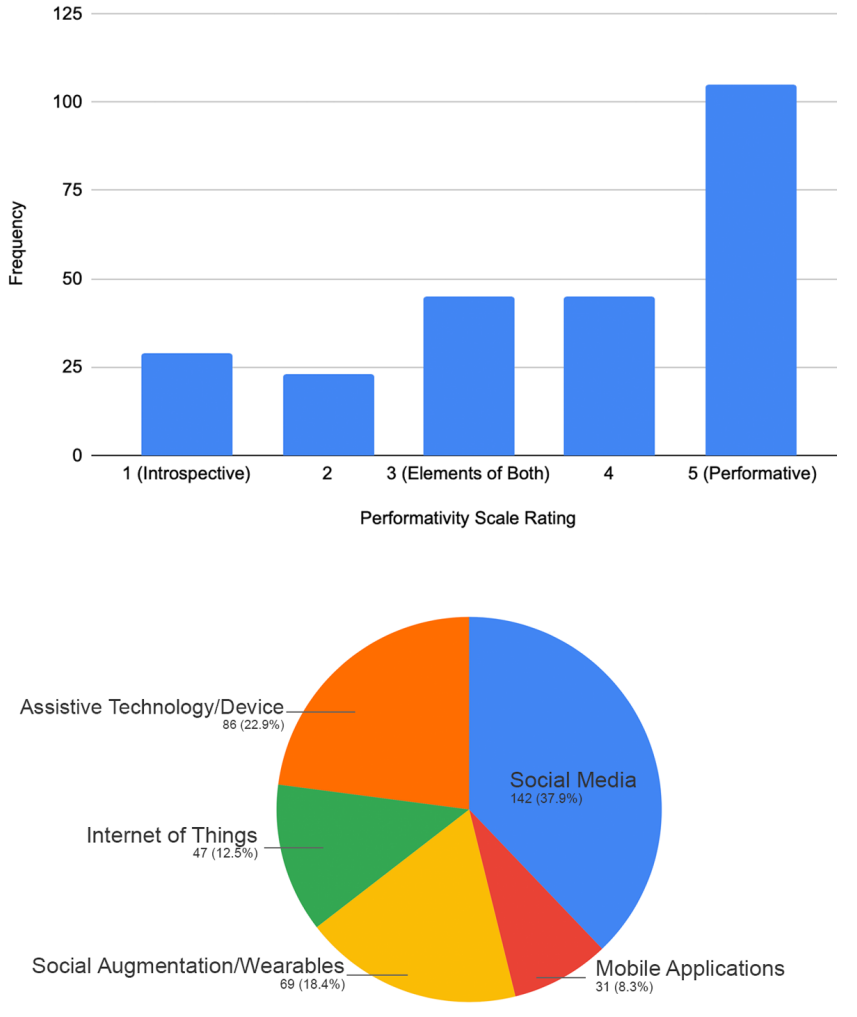

Figure 2: On the top: Frequencies of TikTok videos rated on a 1-5 performativity Likert scale and on the bottom: a pie chart depicting the frequencies of which mediums each video inspired

the play potentials in each of the videos. The frequency of each medium is displayed in the pie chart in the bottom of Figure 2.

\subsection{Everyday Theatrical Life Sketches}

Videos that showcased dramatic performances of "A day in the life of a person with a disability" were categorized into the Everyday Theatrical Life Sketches theme. Of all the 7 themes, this is the broadest, and many of the videos sorted into this theme are also sorted into many of the themes described below. Within this theme, we saw many types of dramatic performances that featured humor, skits, and reenactments. We saw many similarities in the strategies content creators used to improv methodologies used by actors [69]. In particular, content creators would try to recreate moments that were not initially captured on camera but would enhance or dramatize it to be more appealing for their audience. These moments they tried to recreate did not always go as planned, and sometimes the bloopers created more exciting posts, much like an actor might do during an improv sketch.

In Figure 3, the TikTok screenshot numbered 1 depicts a person confidently navigating their house backward in a wheelchair without looking ${ }^{1}$. The TikTok screenshot numbered 2 in Figure 3 shows a blind person humorously crashing into a basketball board ${ }^{2}$. These examples' juxtaposition is interesting because they depict dramatic reenacted moments that represent extreme levels of ability. The first shows mastery of assistive technology that removes a handicap while the second shows how sight is required to play basketball. In both, the background music was carefully selected-in the first, it creates a confident effect, and in the second, it climaxes to a moment of surprise and humor.

\subsection{Playful Advocacy}

Videos that educate the general population on disability subjects were categorized into the Playful Advocacy theme. The effect of these TikToks was generally lighthearted and creative. For example, many videos described proper social etiquette related to disability that creators prefer, such as avoiding pulling blind people by the hand or tugging at their sleeves when helping them cross the street (Instead, offer your assistance, and they will tell you the best way to guide them).

In Figure 3, the TikTok screenshot numbered 3 shows a person who has quadriplegia explaining how they use a hair tie to hold a fork $^{3}$. The TikTok screenshot numbered 4 in Figure 3 is of a person in a wheelchair singing about their partial paralysis ${ }^{4}$. The creators set up a positive and welcoming environment by smiling and using upbeat sound effects in both posts.

\subsection{Debunking Myths and Stereotypes}

Posts that bring to common light misconceptions of people with disabilities are categorized into the Debunking Myths and Stereotypes theme. These videos are often formatted by illustrating the difference between how the audience thinks people with disabilities complete certain tasks versus how they actually complete tasks. Many feature solutions on how people with different disabilities complete everyday tasks that people without disabilities often would not consider, such as the affordances of different types of wheelchairs. Content in these videos is sometimes exaggerated to make a stronger point $-16.12 \%$ are also categorized as Everyday Theatrical Life Sketches-but most are more serious, and many are responses prompted by disrespectful comments on the platform from trolls.

In Figure 3, the TikTok screenshot numbered 5 shows a drummer with 1 hand using 2 drumsticks ${ }^{5}$. The TikTok screenshot numbered 6 in Figure 3 is a blind person showing the difference between how their audience thinks they pour a glass of water versus how they actually pour it ${ }^{6}$. Both are in response to a misconception that their disability prohibits them from possessing a skill or needing help to complete tasks.

\footnotetext{
${ }^{1}$ https://tinyurl.com/y3jj8jeu

${ }^{2}$ https://tinyurl.com/y6yvkecs

${ }^{3}$ https://tinyurl.com/y4c66299

${ }^{4}$ https://tinyurl.com/y4jt5gfo

${ }^{5}$ https://tinyurl.com/y6lehk9p

${ }^{6} \mathrm{https}: / /$ tinyurl.com/y6hzapoc
} 


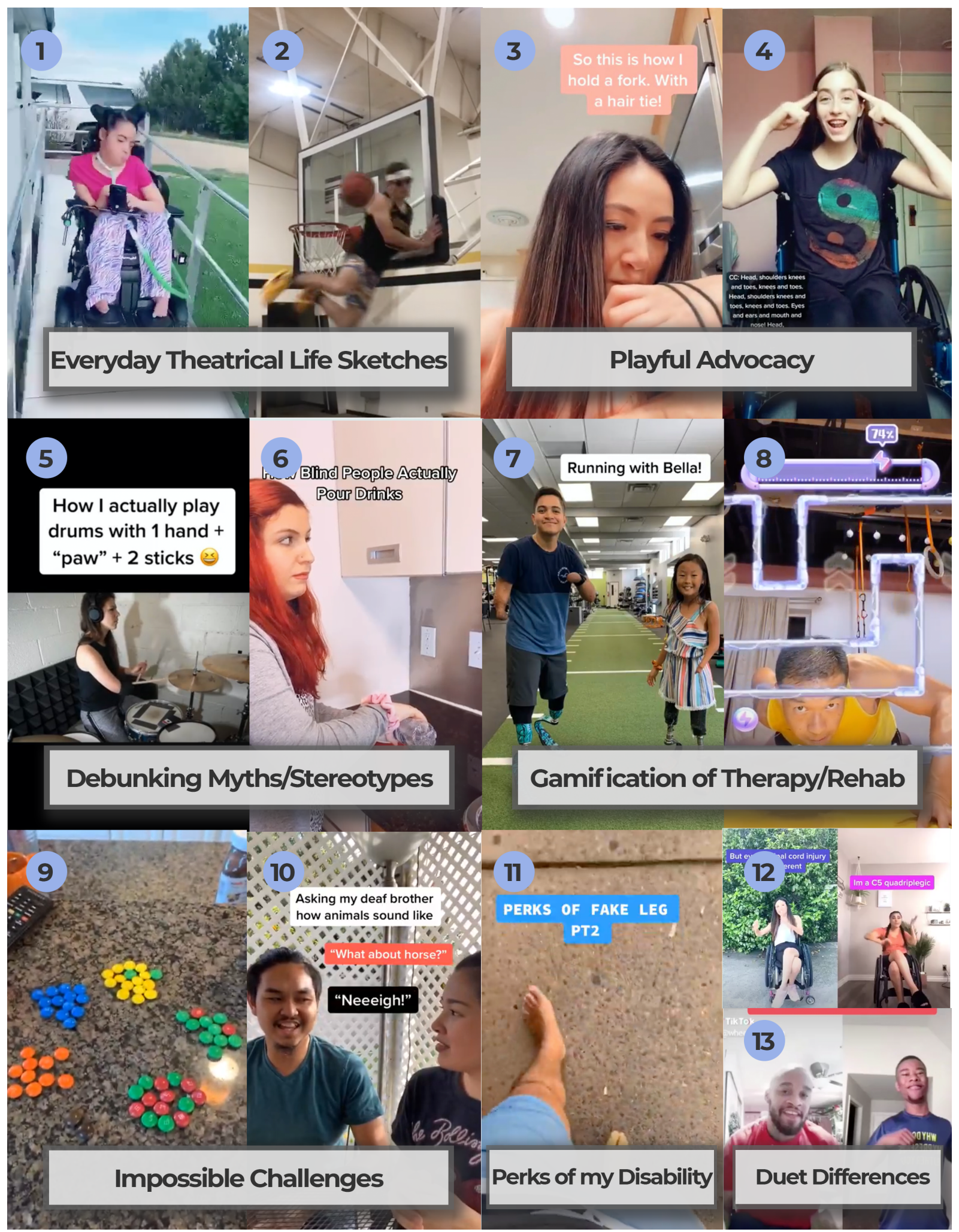

Figure 3: Example TikTok videos organized into each theme labeled by number and described in the text 


\subsection{Gamification of Therapy/Rehabilitation}

TikTok videos that feature games, competition and challenges that motivate completing exercises related to one's disability are categorized into the Gamification of Therapy/Rehabilitation theme. $46.81 \%$ of videos within this theme involve intense physical movement and could directly inspire exergames [77] and physical rehabilitation serious games for health [86]. Dance was mentioned in 41 videos (often due to viral dance challenges) and could be an exciting design opportunity.

In Figure 3, the TikTok screenshot numbered 7 depicts two people with prosthetic legs racing each other ${ }^{7}$. The TikTok screenshot numbered 8 in Figure 3 shows a person with Parkinson's using a screen overlay filter to navigate a digital maze in a 1-handed planking position controlling a dot with their movement to build strength and work through tremors ${ }^{8}$. The first video represents a play potential that has not yet been augmented by technology. In contrast, the second showcases a play potential that has already been augmented, but could be improved with further iteration to include scoring, leader boards, social challenges, maps with dynamic difficulty, sensors, or badges. People naturally appropriate tech, such as camera filters, for their own goals that might not be the original use case, such as turning a maze game into an exergame. Designing playful tech with flexible affordances can lead to novel uses cases that can improve our well-being.

\subsection{Impossible Challenges}

Posts that showcase people with disabilities attempting to complete tasks that they physically cannot do without tools or assistance are categorized into the Impossible Challenges theme. What is interesting about these videos is that the demonstrations can potentially educate the general population through empathy [52]. They showcase how disabilities can impair someone and provide a virtual sensitizing exercise by supplying outsiders with experiences that allow them to adopt the perspective of someone with a disability supporting mine/thine strategies [26]. The public perception of disability can be improved through exposure and mutual understanding. Work in this area has the potential to motivate policy that provides reasonable access and lessens discrimination toward people with disabilities [46].

In Figure 3, the TikTok screenshot numbered 9 depicts a person who is colorblind sorting candy by color 9 . The TikTok screenshot numbered 10 in Figure 3 shows a deaf person trying to produce animal noises such as a pig's oink or a cat's meow-a true challenge given they have never heard the sound or able to hear their own imitation ${ }^{10}$. These videos might spark an education around disabilities by prompting questions such as "If you cannot see red and green, how do you drive?" or "How do deaf people learn to pronounce words?"-which people did ask in the comments. These are important conversations.

\footnotetext{
${ }^{7}$ https://tinyurl.com/yxtd5yyn

${ }^{8}$ https://tinyurl.com/y3heaven

${ }^{9}$ https://tinyurl.com/y4sz66xz

${ }^{10}$ https://tinyurl.com/y3oso8n2
}

\subsection{Perks of My Disability}

Sometimes our disabilities can give us superpowers. There is a common misconception that disability is the opposite of ability [46], but a disability is a physical or mental condition that affects a person's movements, senses, or activities. The Perks of My Disability theme holds content that amplifies moments that creators feel empowered and more able than those without their disability. The example numbered 11 in Figure 3 shows a person using their prosthetic foot on the hot pavement to give their other foot a break from the heat ${ }^{11}$. Another humorous example includes a video of an amputee detaching her prosthetic during a game of Twister to gain an advantage ${ }^{12}$. As a society, we should celebrate perks of disabilities, such as the unique visuospatial abilities of people with autism [59]. Well-designed assistive technology has the potential to empower people with disabilities uniquely.

\subsection{Duet Differences}

By definition, the affordances of technology shape how people use it [40] and appropriate it, which became necessary for our analysis because people with disabilities used TikTok's "Duet" feature to juxtapose their own video with others'. There are two primary ways people with disabilities used the "Duet" feature: to share their realtime reactions to another post or to explain how they do an activity differently from someone doing the same activity in another post. When posts used the duet feature to highlight something about the creator's disability, the video was categorized into the Duet Differences theme. There are no such things as groups on TikTok, and creators use the "Duet" feature to create communities within the social network. In Figure 3, the TikTok screenshot numbered 12 depicts two people who are $\mathrm{C} 5$ quadriplegics comparing how the same diagnosis affects their dancing abilities ${ }^{13}$. The TikTok screenshot numbered 13 in Figure 3 shows two people with Cerebral Palsy completing a dance challenge to raise awareness about their disability. In both of these examples, people with the same disability highlight their differences, while at the same time fostering a community of education and growth around disability awareness on TikTok.

\section{DESIGN CONCEPT CATALOG}

We created 20 design concepts based on play potentials found from scraping TikTok for playful videos featuring content creators with disabilities and share 8 of our favorite concepts in this paper. These concepts are meant to illustrate one method of engaging populations with disabilities, to inspire possible future technologies, and to advocate for inclusive and accessible general technology design. Our goal was to create a variety of ideas, so we did not constrain ourselves to the limitations of current technology or a particular platform. We also did not limit our ideas to only accessible technology or only assistive technology, though some of the concepts indeed could be described as accessible or assistive. Our work is generative-while some of our concepts could feasibly be prototyped with today's technology, iterated on, and evaluated by stakeholders, the purpose of this work is not to build and evaluate

\footnotetext{
$\overline{{ }^{11} \text { https://tinyurl.com/yxejuxtk }}$

${ }^{12}$ https://tinyurl.com/y58zn2xg

${ }^{13}$ https://tinyurl.com/y2emowms
} 


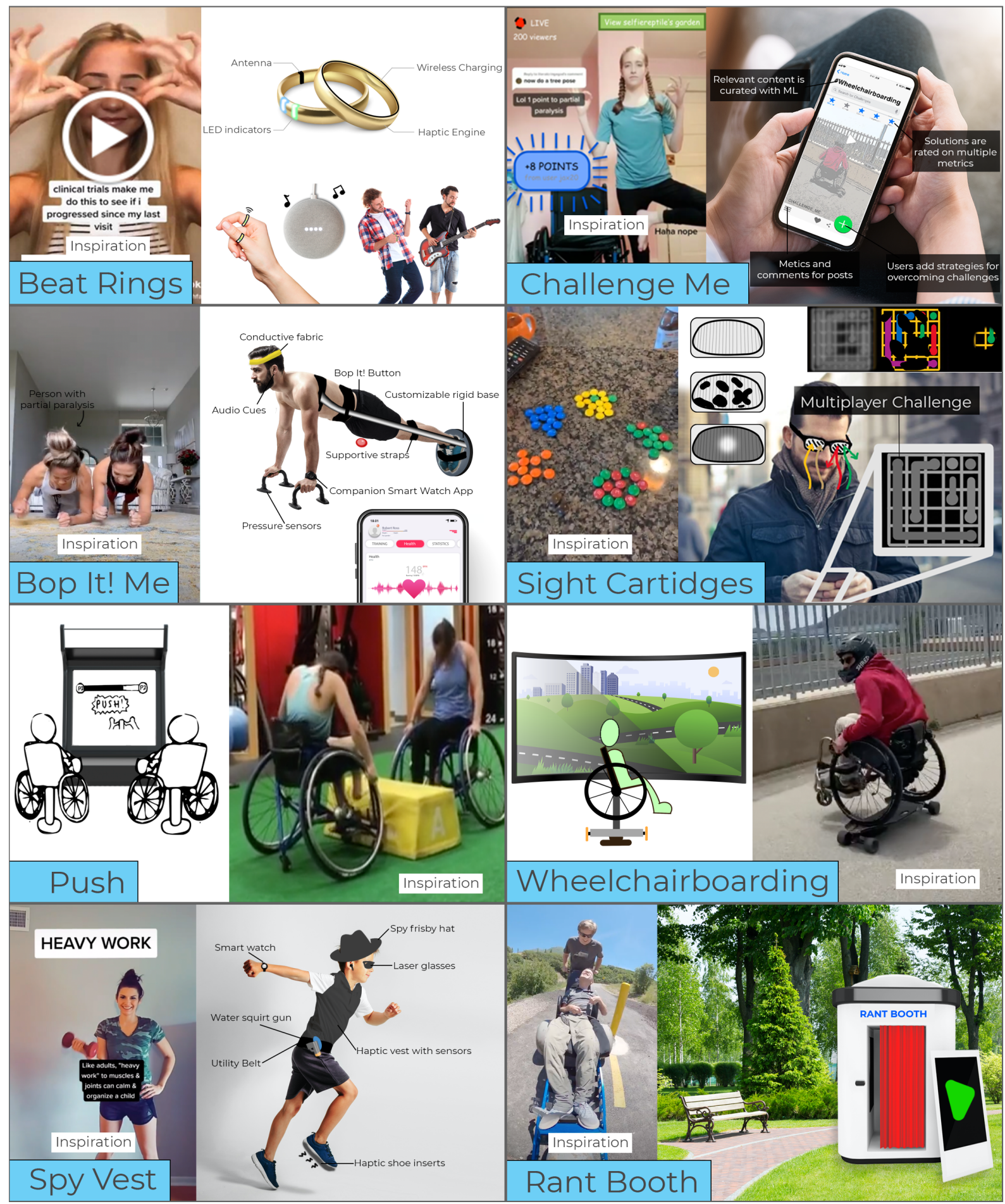

Figure 4: Compilation of our catalog of design concept sketches that are individually described in the text 
a system, it is to envision and speculate possible future designs [8] and to add to a larger body of work calling for disability-inclusive design [18, 42, 70, 84, 85]. In this section, we describe a subset of our design concepts and reflect on the unique elements of each, but provide a broader discussion as part of the Discussion section of this paper. Table 2 provides an overview of the concepts, including the populations they could be relevant for, the technology mediums that might be interesting to implement each of the designs in, which of the themes described above the design supports, whether or not the design has the potential to increase the visibility of people with disabilities in society, and whether or not the technology has the potential to be assistive to people with disabilities.

\subsection{BeatRings}

Beat Rings was inspired by a person with a neuromuscular disease called Friedreich Ataxia tracking her hand coordination progress by tapping her fingers to a viral challenge called the "\#transitionChallenge" that requires a person to tap each hand at a different rate 14. People, regardless of their disabilities status, participated in the viral TikTok challenge, and we hypothesize that Beat Rings could be enjoyable broadly. The sketch in Figure 4 shows the original post that inspired this design, a mockup of Beat Rings, and how they might be used in a social setting. We envision Beat Rings to be unobtrusive rings worn on fingers and embedded with accelerometers to detect tapping and wireless communication abilities to be able to communicate with smart speakers and other smart devices. Users could wear as many rings as they wanted on whichever fingers they want to record actively. Each ring can be assigned an instrument or a riff, and users can play by tapping their fingers to their thumb or tapping on any other surface. Users can create Guitar Hero-type challenges to battle with friends or jam together without needing to know how to play an instrument.

\subsection{Challenge Me App}

The Challenge Me App is a social media concept that fosters communities of people with disabilities to skillshare, similar to DIY culture. It was inspired by many play potentials found within the Everyday Theatrical Life Sketches theme. Figure 4 shows one of the posts that inspired the design and a mockup of what the app could look like. The app would foster creativity by leveraging our collective nature to develop the best solution to a specific challenge. For example, opening a door without arms could be a challenge that the app collects solutions that can be up-voted for dimensions such as originality, creativity, cost-effectiveness, complexity, delivery, accessibility, dependability, and independence. The app has the potential to foster innovation within the community, foster community building, skillshare, and inspire better assistive technology.

\subsection{Bop It! Me}

Bop It toys are a line of audio games that issue a random series of pre-recorded audio commands to press buttons, pull handles, twist cranks, spin wheels, and flick switches on the toy quickly as the pace of the game and the player's score increases. Bop It! $M e$ is a design concept inspired by a TikTok viral dance challenge where a person with partial paralysis completed the dance with

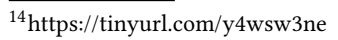

the help of another who moved their legs for them. The concept idea, shown in Figure 4, features rigid body braces that would keep a person (paralyzed or not) in a plank position and from moving their lower body. The device has strategically placed Bop It!-style sensors such as conductive fabric, a headband for wiping one's brow, a button to bop one's hip, a sensor on a rotating base for one to "twist" or rollover, as well as an array of accelerometers to detect exercises such as push-ups. The device could support independent high scores, instructions for high-intensity, short-interval exercises, and exergames. It could be modular to customize sensors' placement and control how physically demanding the experience is. The frame could also be modular and provide rigid support for different ability levels and challenge levels.

\subsection{Sight Cartridges}

Sight Cartridges is inspired by TikToks showcasing glasses that allow people who are colorblind to see color for the first time ${ }^{15}$, empathy tools for simulating various vision impairments [10], and the asymmetrical virtual reality game titled Keep Talking and Nobody explodes [2]. The premise of this concept, shown in Figure 4 is a game where players collaborate to solve visual challenges wearing glasses that afford them different visual privileges, such as being able to see color, to see a wide field of view without center clarity, or to see some areas sharply without a wide field of view. The glasses would have interchangeable cartridges that simulate different visual impairments to be used in the game and could also be used outside the game as empathy tools. Players with visual impairments would not need to use the glasses. The game itself could have various themes such as nanobots with different abilities working together to find their target, alien creatures adapting to a new planet with multiple stars, or coming to terms in a new life inside the Matrix with malfunctioning brain-computer interfaces.

\subsection{Push!}

Push! is a design concept inspired by a TikTok showcasing two wheelchair users pushing against a yellow block in a strength competition where they try to move the block into the other player's territory similar to Tug-of-War ${ }^{16}$, shown in Figure 4. This design concept would be found in an arcade where sensors are used to keep high scores, and the game could feature narratives such as personifying a bulldozer, racing as a locomotive, or spinning the wheels independently to generate music. This design concept has the potential to increase the visibility of disabilities in a safe public space, allow people to experience a wheelchair, and compete against actual wheelchair users (who would most likely win) on an equal playing field.

\subsection{Wheelchairboarding}

Wheelchairboarding is based directly on a TikTok video showcasing a person in a wheelchair placed on top of a skateboard speeding down the road laterally and steering by tilting forward and backward on the balanced wheelchair ${ }^{17}$. This design concept is another arcade game similar to other racing arcade games that simulate

\footnotetext{
$\overline{{ }^{15} \text { https://tinyurl.com/y5wqg2cb }}$

${ }^{16}$ https://tinyurl.com/y5p7xeky

${ }^{17}$ https://tinyurl.com/y2hu5gu4
} 
Table 2: Overview of design concepts

\begin{tabular}{|c|c|c|c|c|c|}
\hline Name & Target Population & Medium & Themes & Visibility Potential & Therapy Potential \\
\hline \multirow[t]{3}{*}{ Beat Rings } & General Population & Tangible & Theater Sketches & \multirow[t]{3}{*}{ Yes } & \multirow[t]{3}{*}{ Yes } \\
\hline & Physical Impairments & Audio & Gamification & & \\
\hline & Sensory Tool & & Duet Differences & & \\
\hline \multirow[t]{3}{*}{ Challenge Me } & People with Disabilities & Screens & Debunking Stereotypes & \multirow[t]{3}{*}{ Yes } & \multirow[t]{3}{*}{ Yes } \\
\hline & General Population & & Impossible Challenges & & \\
\hline & & & Duet Differences & & \\
\hline \multirow[t]{2}{*}{ BopIt! Me } & General Population & Wearables & Gamification & \multirow[t]{2}{*}{ Yes } & \multirow[t]{2}{*}{ Yes } \\
\hline & Physical Impairments & & Impossible Challenges & & \\
\hline \multirow[t]{4}{*}{ Sight Cartridges } & General Population & Wearables & Playful Advocacy & \multirow[t]{4}{*}{ Yes } & \multirow[t]{4}{*}{ No } \\
\hline & & Mixed Reality & Debunking Stereotypes & & \\
\hline & & & Impossible Challenges & & \\
\hline & & & Perks of my Disability & & \\
\hline \multirow[t]{2}{*}{ Push! } & General Population & Arcade & Gamification & \multirow[t]{2}{*}{ Yes } & \multirow[t]{2}{*}{ Yes } \\
\hline & Wheelchair Users & & Perks of My Disability & & \\
\hline \multirow[t]{4}{*}{ Wheelchairboarding } & General Population & Arcade & Theater Sketches & \multirow[t]{4}{*}{ Yes } & \multirow[t]{4}{*}{ No } \\
\hline & Wheelchair Users & & Playful Advocacy & & \\
\hline & & & Debunking Stereotypes & & \\
\hline & & & Perks of My Disability & & \\
\hline \multirow[t]{2}{*}{ Spy Vest } & General Population & Wearables & Theater Sketches & \multirow[t]{2}{*}{ No } & \multirow[t]{2}{*}{ Yes } \\
\hline & & Internet of Things & Gamification & & \\
\hline \multirow[t]{3}{*}{ Rant Booth } & People with Disabilities & Tangible & Playful Advocacy & \multirow[t]{3}{*}{ Yes } & \multirow[t]{3}{*}{ No } \\
\hline & General Population & Internet of Things & Debunking Stereotypes & & \\
\hline & & & Impossible Challenges & & \\
\hline
\end{tabular}

various vehicles, shown in Figure 4. This game has the potential to have the same visibility and empathy benefits described in Push!, but also has the potential to provide a safe space for wheelchair users to practice skateboarding.

\subsection{Spy Vest}

Spy Vest is inspired by a series of TikToks from mothers sharing ideas and strategies for raising children with Autism Spectrum Disorder, such as finishing chores before getting the WiFi password or creating soothing sensory experiences. Many of the mothers expressed difficulty motivating their children to get physical activity. Spy Vest, shown in Figure 4 is a wearable design concept featuring earbuds, accelerometers, haptic feedback, and a water squirt gun that can be enabled and disabled by the tech. The vest feeds secret missions to the child through the earbuds and uses the sensors to validate that the exercises are being performed. As a reward, the child gets a final puzzle that unlocks the WiFi and allows them to shoot water at their parents and siblings.

\subsection{Rant Booth}

Rant Booth is inspired by TikTok videos featuring people with disabilities ranting about the public infrastructure that handicaps them and from videos where content creators find strangers in public spaces and interview them about their lives and donate crowdsourced funds to those in need. Rant Booth, shown in Figure 4, is a private booth located in accessible public spaces that playfully lures people in and prompts them to rant about challenges in their community. The booth would create a montage of humorous themed animations and altered voices using machine learning sent to policy-makers and local officials, prompting them to invest in a more universally accessible infrastructure based on community feedback.

\section{DISCUSSION}

This paper's primary purpose is to advocate for the design of playful technology for and to build designer empathy for people with disabilities. Technology development should include people with disabilities as stakeholders, and technology designed with and for people with disabilities does not always need to be serious. "Disability is a natural part of the human condition resulting from that spectrum-and will touch most of us at one time or another in our lives. The goal is not to fixate on, overreact to, or engage in stereotypes about such differences, but to take them into account and allow for reasonable accommodation for individual abilities and impairments that will permit equal participation"[46]. Our first research question, RQ1 (What play potentials exist from scraping content on TikTok from creators with disabilities?), involved unearthing playful content from people with disabilities on TikTok. There were three strategies available to us for finding these posts: 1) searching keywords in the form of hashtags, 2) "favoriting" videos that met our criteria to train the "For You" page's curation algorithm, and 3) following content creators with disabilities. The process created an interesting feedback loop between us and the curation algorithm that likely uses machine learning. In some ways, in our work, the algorithm took a research assistant's role because it sought out data for our study. The massive scale biases and assumptions the algorithms foster likely impacted our work. There have been numerous news articles that describe how the TikTok algorithm suppresses the voices of those with disabilities, most likely due to how the general population interacts with content from people with disabilities. Massive-scale interactions with people with disabilities may 
have the potential to train the curation algorithms to show content from people with disabilities to the general population more often, creating more visibility.

Our second research question, RQ2 (What themes emerge from an analysis of scraped play potentials and how does the TikTok platform influence the results?), involved analyzing the TikTok posts we logged to sort them into 7 emerging themes, highlight exemplars, rate the level of "performativity", and tagging technological mediums that would be interesting to design for. We found that content on TikTok is generally performative, exaggerated, and dramatized, indicating that our design concepts are more likely to elicit these types of experiences. TikTok is likely a less appropriate platform to find play potentials that inspire more introspective designs. Many of the play potentials we found are about how people with disabilities are playful with social media-that is, they involve social media-which is very different from being playful without social media, possibly limiting the scope and applicability of our themes.

Our third research question, RQ3 (What kinds of designs can the scraped play potentials inspire and how might these designs affect society?) began with using the exemplars to inspire design concepts. Our 8 designs concepts are just that-concepts-they were inspired by people with disabilities and contain expertise from formally trained designers, but they are jumping-off points for future work in their current state. Soon, when technology and sensors' abilities can support these designs, the concepts would need to be evaluated, vetted, and iterated on with stakeholders in the target population. The design catalog can also serve as a conversation piece for facilitating discussions around speculative futures [8]. We begin this conversation by speculating on some of these potential future implications in the next section.

\subsection{Possible Implications of Design Concepts}

A common theme in two of our design concepts was using arcades as a safe space to facilitate disability visibility, understanding, and empathy. Arcades are shared spaces where people of many ages, genders, and cultures come together to play. Arcade games can be accessible to those with various disabilities and can host games that emulate assistive technology and devices for everyone to experience. These games can allow people with and without disabilities to play together, fostering relationships and friendships.

Many of our concepts could be enjoyed by both people with and without disabilities, such as the Beat Rings and Spy Vest-and could be played socially by peers regardless of their disability status. One key design goal for possible future implementations of these concepts is modular designs that are flexible to accommodate many use cases and ability levels. For example, the Bop It! Me concept uses a frame that allows different rigid supports for those with movement impairments. People are naturally adept at leveraging and appropriating affordances to accommodate their needs. For example, most creators with disabilities used the "Stickers" video editing feature in TikTok to create closed captions for their videos even though it does not natively support them. Some of our concepts are designed to be used exclusively by people with disabilities, such as Challenge Me, which fosters community building and skillsharing.
Interestingly, there are many similarities to the enhance the play step proposed in the Situated Play Design methodology [3] in the enhancements content creators made to their performances. Many of the posts were clearly re-enactments, scripted, and dramatized to make them more entertaining. This is similar to enhance the play because content creators made mundane, everyday life more fun, suggesting that people without formal design training and people with disabilities are natural play chasers. Now, with the global epidemic, it is more evident than ever that we need more joy and play in our lives-and content creators are bridging this gap during these stressful times with their heightened and playful performances. Technology design that supports playful performances, as some of our design concepts illustrate (e.g., Beat Rings, Challenge Me, Bop It! Me, Wheelchairboarding, and Spy Vest), should support performers who have disabilities as well. Through continued exposure to designs that support performers with disabilities, our societies may build a greater understanding of disability as a political concept, advocate more for equitable access, and become more inclusive.

When augmenting the play potentials we found, we did not prioritize designs that served pragmatic goals. All of our design concepts have the potential to improve the public image of people with disabilities, foster empathy within communities, and promote play, which is generally beneficial-all of which are the primary motivations of this work. The Rant Booth concept represents a playful technology that could directly influence policy-makers and community leaders who have the power and capital to make a more accessible infrastructure. Based on the nature of the content we scraped on TikTok where people with disabilities often showcased how they deal with their disability, some of our design concepts could be considered assistive technology that could serve a pragmatic purpose. For example, Beat Rings can help people with fine motor impairments, Bop It! Me can help improve stamina, and Push! can improve strength.

Our results confirmed our hypothesis that TikTok is a performative platform where the content tends to be exaggerated, dramatized, and elevated. The emerging themes and the resulting concepts were directly fueled by performative TikTok content, impacting the demographics that might be interested in the futures our designs may support.

\subsection{Limitations}

Our work's most prominent limitation is that we did not directly engage with people with disabilities-we indirectly scraped their content from TikTok. People with disabilities have not yet evaluated our concepts, and when appropriate, the specific target populations they could impact. In this work, our priority was to maintain a safe distance from these populations who often are at higher risk of COVID19, and to refrain from placing any extra burdens on them during these stressful times, while still gaining design inspiration and insight. The goal of our work was to generate concepts that might inspire future technology, using existing content from these communities to inspire technology design, and to support our research agenda that general everyday technology should be playful, 
inclusive, and sometimes, non-utilitarian. Instead of a formal evaluation, we engaged in speculative design [8] practices to evaluate the potential of the design catalog.

Our design concepts are intentionally vague in many ways. We did not design every interaction, affordance, and detail. We designed speculative design concepts that can serve as inspiration for future technology or as discussion probes. When it comes time to bring designs to fruition, a participatory approach should be used and include people with disabilities. Our design concepts were internlead and directly inspired by exemplar TikToks they selected. More experienced designers may use our data set to generate novel ideas based more broadly on our emerging themes and their expertise.

TikTok has been and may continue to be banned in many countries, but the lessons learned here may be generalizable beyond this particular social media platform. There are many social media platforms that feature playful video content from people with disabilities, including Youtube, Vimeo, Instagram, Facebook, and Twitter. Much of what is discussed in this paper may be generalizable to chasing play on those platforms.

\subsection{Future Work}

Much of the scraping, brainstorming, and design work was completed by high school students (who are also authors of this paper), participating in an 8-week summer internship. From a methodological and pedagogical standpoint, there are many exciting insights documented in their logs and reflections and our experience doing research-through-design work with them. The internship also served as an entry point into empathy for people with disabilities. There was much growth and learning with each of the interns during the 8-week internship, and we would like to share these insights with the ASSETS community (the premier conference on accessible computing). We want to work with stakeholders on bringing some of these concepts to fruition for further study. As we continue researching other platforms, we are interested in formulating suggestions to social media companies to better support people with disabilities to improve their products and make them more enjoyable for populations with disabilities.

\section{CONCLUSION}

We collectively scraped content on TikTok from content creators with disabilities for 100 hours ( 5 interns for an hour a day, 5 days a week, for 4 weeks), resulting in 285 video posts containing play potentials [3] (RQ1). We analyzed and organized the posts into 7 emerging themes: Everyday Theatrical Life Sketches, Playful Advocacy, Debunking Myths and Stereotypes, Gamification of Therapy/Rehab, Impossible Challenges, Perks of My Disability, and Duet Differences (RQ2). Finally, we created a catalog of 8 design concepts inspired by some of the play potentials and speculate on how they could inform the design of future playful technology that supports people with disabilities (RQ3). We found that content creators are natural performers and play chasers. We discussed how our concepts have the potential to inspire designs that can facilitate disability visibility, understanding, and empathy-and the importance of this potential impact on society cannot be overstated. Our contributions add to a larger body of work advocating for the inclusion of people with disabilities in the design process. We provide the first data set of TikTok posts focused exclusively on content creators with disabilities being playful using the Situated Play Design methodology.

\section{ACKNOWLEDGMENTS}

Most importantly, we would like to thank all of the content creators on TikTok who inspired us and enabled this work. We appreciate the Science Internship Program and all of the participating interns who impressed us with their skills and eagerness to learn. We are grateful to the TikTok platform for enabling diverse content creators. Finally, we appreciate the quality feedback from our reviewers that strengthened the presentation of our work.

\section{REFERENCES}

[1] Allen, Burk, and Davis. 2006. Academic Data Collection in Electronic Environments: Defining Acceptable Use of Internet Resources. MIS Quarterly 30, 3 (2006), 599. https://doi.org/10.2307/25148741

[2] Allen Allen Pestaluky, Ben Kane, and Brian Fetter. 2015. Keep Talking Nobody Explodes.

[3] Ferran Altarriba Bertran, Jared Duval, Katherine Isbister, Danielle Wilde, Elena Márquez Segura, Oscar Garcia Pañella, and Laia Badal León. 2019. Chasing Play Potentials in Food Culture to Inspire Technology Design. In Extended Abstracts of the Annual Symposium on Computer-Human Interaction in Play Companion Extended Abstracts (CHI PLAY' 19 Extended Abstracts). Association for Computing Machinery, New York, NY, USA, 829-834. https://doi.org/10.1145/3341215. 3349586 event-place: Barcelona, Spain.

[4] Ferran Altarriba Bertran, Elena Márquez Segura, Jared Duval, and Katherine Isbister. 2019. Chasing Play Potentials: Towards an Increasingly Situated and Emergent Approach to Everyday Play Design. In Proceedings of the 2019 on Designing Interactive Systems Conference (DIS '19). Association for Computing Machinery, New York, NY, USA, 1265-1277. https://doi.org/10.1145/3322276. 3322325 event-place: San Diego, CA, USA.

[5] Ferran Altarriba Bertran, Elena Márquez Segura, Jared Duval, and Katherine Isbister. 2019. Designing for Play That Permeates Everyday Life: Towards New Methods for Situated Play Design. In Proceedings of the Halfway to the Future Symposium 2019 (HTTF 2019). Association for Computing Machinery, New York, NY, USA, 4. https://doi.org/10.1145/3363384.3363400 event-place: Nottingham, United Kingdom.

[6] Thiru M. Annaswamy, Monica Verduzco-Gutierrez, and Lex Frieden. 2020. Telemedicine barriers and challenges for persons with disabilities: Covid-19 and beyond. Disability and Health fournal 13, 4 (July 2020), 4. https://doi.org/10. 1016/j.dhjo.2020.100973

[7] Richard Armitage and Laura B. Nellums. 2020. The COVID-19 response must be disability inclusive. The Lancet Public Health 5, 5 (May 2020), e257. https: //doi.org/10.1016/S2468-2667(20)30076-1 Publisher: Elsevier.

[8] James Auger. 2013. Speculative design: crafting the speculation. Digital Creativity 24, 1 (2013), 11-35. Publisher: Taylor \& Francis.

[9] Elizabeth M Badley. 1995. The genesis of handicap: definition, models of disablement, and role of external factors. Disability and rehabilitation 17, 2 (1995), 53-62. Publisher: Taylor \& Francis.

[10] Jialiang Bai, Zhefan Yu, Fengjie Zhang, and Yeshuai Cheng. 2019. Empathy Tool Design-Eye Disease Simulator Based on Mixed-Reality Technology. In HCI International 2019 - Posters, Constantine Stephanidis (Ed.). Springer International Publishing, Cham, 235-242.

[11] Elizabeth H. Bassett and Kate O'Riordan. 2002. Ethics of Internet research: Contesting the human subjects research model. Ethics and Information Technology 4, 3 (2002), 233-247. https://doi.org/10.1023/A:1021319125207

[12] Buster Benson. 2015. A gameful mind. In The gameful world : approaches, issues, applications. The MIT Press, Cambridge.

[13] Erling Bjögvinsson, Pelle Ehn, and Per-Anders Hillgren. 2012. Design things and design thinking: Contemporary participatory design challenges. Design issues 28, 3 (2012), 101-116.

[14] Sharon Boller and Karl Kapp. 2017. Play to learn: Everything you need to know about designing effective learning games. Association for Talent Development, Alexandria, VA.

[15] Sue Bratton and Dee Ray. 2000. What the research shows about play therapy. International fournal of Play Therapy 9, 1 (2000), 47.

[16] Sue C Bratton, Dee Ray, Tammy Rhine, and Leslie Jones. 2005. The efficacy of play therapy with children: A meta-analytic review of treatment outcomes. Professional Psychology: Research and Practice 36, 4 (2005), 376.

[17] Stuart Brown. 2009. Play: How it shapes the brain, opens the imagination, and invigorates the soul. Avery/Penguin Group USA, New York, NY, USA, New 
York, NY. https://www.proquest.com/books/play-how-shapes-brain-opensimagination/docview/622167753/se-2?accountid=14523

[18] Susanne Bødker and Morten Kyng. 2018. Participatory design that matters-Facing the big issues. ACM Transactions on Computer-Human Interaction (TOCHI) 25, 1 (2018), 4.

[19] Roger Caillois. 2001. Man, play, and games. University of Illinois Press, USA Chicago, Illinois.

[20] Sandra L Calvert. 2011. Cognitive effects of video games. In Handbook of computer game studies. MIT Press, Cambridge, Mass, 125-131. Publisher: MIT Press Cambridge, MA.

[21] James I Charlton. 2000. Nothing about us without us: Disability oppression and empowerment. Univ of California Press, Berkeley, CA USA.

[22] Mihaly Csikszentmihalyi, Sami Abuhamdeh, and Jeanne Nakamura. 2014. Flow. In Flow and the foundations of positive psychology. Springer, New York, NY, USA, 227-238.

[23] Nonny De la Peña, Peggy Weil, Joan Llobera, Elias Giannopoulos, Ausiàs Pomés Bernhard Spanlang, Doron Friedman, Maria V Sanchez-Vives, and Mel Slater. 2010 Immersive journalism: immersive virtual reality for the first-person experience of news. Presence: Teleoperators and virtual environments 19, 4 (2010), 291-301.

[24] Katherine Deibel. 2013. A convenient heuristic model for understanding assistive technology adoption. In Proceedings of the 15th International ACM SIGACCESS Conference on Computers and Accessibility. Association for Computing Machinery, New York, NY, USA, 1-2. https://doi.org/10.1145/2513383.2513427

[25] Steven Dow, Blair MacIntyre, Jaemin Lee, Christopher Oezbek, Jay David Bolter and Maribeth Gandy. 2005. Wizard of $\mathrm{Oz}$ support throughout an iterative design process. IEEE Pervasive Computing 4, 4 (2005), 18-26.

[26] Dana S Dunn, David J Fisher, and Brittany M Beard. 2012. Revisiting the mine/thine problem: A sensitizing exercise for clinic, classroom, and attributional research. Rehabilitation psychology 57, 2 (2012), 113. Publisher: American Psychological Association.

[27] Jared Duval. 2017. A mobile game system for improving the speech therapy experience. In Proceedings of the 19th International Conference on HumanComputer Interaction with Mobile Devices and Services. ACM, New York, NY, USA, 3. https://doi.org/10.1145/3098279.3119925

[28] Scott G Eberle. 2014. The elements of play: Toward a philosophy and a definition of play. American fournal of Play 6, 2 (2014), 214-233.

[29] Carolyn Ellis, Tony E. Adams, and Arthur P. Bochner. 2011. Autoethnography: An Overview. Historical Social Research / Historische Sozialforschung 36, 4 (138) (2011), 273-290. http://www.jstor.org/stable/23032294 Publisher: GESIS - LeibnizInstitute for the Social Sciences, Center for Historical Social Research.

[30] Aviv Elor, Sri Kurniawan, and Mircea Teodorescu. 2018. Towards an Immersive Virtual Reality Game for Smarter Post-Stroke Rehabilitation. In 2018 IEEE International Conference on Smart Computing (SMARTCOMP). IEEE, New York, NY, USA, 219-225.

[31] Aviv Elor, Mircea Teodorescu, and Sri Kurniawan. 2018. Project Star Catcher A Novel Immersive Virtual Reality Experience for Upper Limb Rehabilitation ACM Transactions on Accessible Computing 11, 4 (Nov. 2018), 1-25. https://doi. org $/ 10.1145 / 3265755$

[32] Tim Freeman. 2006. 'Best practice'in focus group research: making sense of different views. Fournal of advanced nursing 56, 5 (2006), 491-497. Publisher Wiley Online Library.

[33] R. Garris, R. Ahlers, and J. E. Driskell. 2002. Games, Motivation, and Learning: A Research and Practice Model. Simulation \& Gaming 33, 4 (Dec. 2002), 441-467. https://doi.org/10.1177/1046878102238607

[34] Bill Gaver, Tony Dunne, and Elena Pacenti. 1999. Design: cultural probes. interactions 6, 1 (1999), 21-29. Publisher: ACM New York, NY, USA.

[35] Kathrin Gerling, Kieran Hicks, Michael Kalyn, Adam Evans, and Conor Linehan 2016. Designing Movement-based Play With Young People Using Powered Wheelchairs. In Proceedings of the 2016 CHI Conference on Human Factors in Computing Systems. ACM, San Jose California USA, 4447-4458. https://doi.org/ $10.1145 / 2858036.2858070$

[36] Kathrin Maria Gerling, Regan L. Mandryk, Max Valentin Birk, Matthew Miller, and Rita Orji. 2014. The effects of embodied persuasive games on player attitudes toward people using wheelchairs. In Proceedings of the SIGCHI Conference on Human Factors in Computing Systems. ACM, Toronto Ontario Canada, 3413-3422. https://doi.org/10.1145/2556288.2556962

[37] Barrie Gunter. 2005. Psychological effects of video games. In Handbook of computer game studies. MIT Press, Cambridge, Mass, 145-60.

[38] Sven Ove Hansson. 2007. The ethics of enabling technology. Cambridge $Q$. Healthcare Ethics 16 (2007), 257. Publisher: HeinOnline.

[39] Lynne Harkness and Anita C Bundy. 2001. The test of playfulness and children with physical disabilities. The Occupational Therapy fournal of Research 21, 2 (2001), 73-89. Publisher: SAGE Publications Sage CA: Los Angeles, CA.

[40] Rex Hartson. 2003. Cognitive, physical, sensory, and functional affordances in interaction design. Behaviour \& information technology 22, 5 (2003), 315-338. Publisher: Taylor \& Francis.

[41] Linda L Hestenes and Deborah E Carroll. 2000. The play interactions of young children with and without disabilities: Individual and environmental influences.
Early Childhood Research Quarterly 15, 2 (Jan. 2000), 229-246. https://doi.org/10. 1016/S0885-2006(00)00052-1

[42] Harald Holone and Jo Herstad. 2013. Three tensions in participatory design for inclusion. In Proceedings of the SIGCHI Conference on Human Factors in Computing Systems. ACM, Paris France, 2903-2906. https://doi.org/10.1145/2470654.2481401

[43] Steve Hoppes, Tim Wilcox, and Greta Graham. 2001. Meanings of play for older adults. Physical \& Occupational Therapy in Geriatrics 18, 3 (2001), 57-68.

[44] Johan H Huizinga. 1980. Homo ludens: a study of the play-element in culture. Routledge, Abingdon, United Kingdom. OCLC: 948679555.

[45] Katherine Isbister. 2016. How games move us: emotion by design. MIT Press, Cambridge, MA.

[46] Mary Johnson. 2007. Before its time: Public perception of disability rights, the Americans with disabilities act, and the future of access and accommodation. Wash. UfL \& Pol'y 23 (2007), 121. Publisher: HeinOnline.

[47] Shaun K. Kane, Chandrika Jayant, Jacob O. Wobbrock, and Richard E. Ladner. 2009. Freedom to roam: a study of mobile device adoption and accessibility for people with visual and motor disabilities. In Proceeding of the eleventh international ACM SIGACCESS conference on Computers and accessibility - ASSETS '09. ACM Press, Pittsburgh, Pennsylvania, USA, 115. https://doi.org/10.1145/1639642.1639663

[48] John H. Kerr and Michael J. Apter. 1991. Adult play : a reversal theory approach. Swets \& Zeitlinger, Amsterdam.

[49] Anja Kintsch and Rogerio Depaula. 2002. A framework for the adoption of Assistive Technology. In in ASSETS 2002. ACM Press, New York, NY, USA, 1-10.

[50] Robert Kozinets. 2019. Ethics. In Netnography: the essential guide to qualitative social media research (3rd edition ed.). SAGE Publications, Thousand Oaks, CA, 127-159.

[51] Robert Kozinets. 2019. Netnography: the essential guide to qualitative social media research (3rd edition ed.). SAGE Publications, Thousand Oaks, CA.

[52] Charles Adam Laffiteau. 2020. Employing Empathy: Using Video Simulations as an Intervention to Educate Social Work Students. PhD Thesis. University of Arkansas.

[53] María López Hernández. 2020. Healthcare Gamification - Serious Game About COVID-19; Stay at home. Master's thesis. Malmö University, Faculty of Culture and Society (KS). Backup Publisher: Malmö University, Faculty of Culture and Society (KS)

[54] Alex Makedon. 1984. Playful gaming. Simulation \& games 15, 1 (1984), 25-64.

[55] Jane McGonigal. 2004. Alternate Reality Gaming: Life imitates ARG. http://avantgame.com/McGonigal_ARG_Austin\%20Game\%20Conference_ Oct2005.pdf

[56] Jane McGonigal. 2015. Superbetter: a revolutionary approach to getting stronger, happier, braver, and more resilient. Penguin, City of Westminster, London, England. OCLC: 1060759600.

[57] Edward F. McQuarrie, Jessica Miller, and Barbara J. Phillips. 2013. The Megaphone Effect: Taste and Audience in Fashion Blogging. Fournal of Consumer Research 40, 1 (June 2013), 136-158. https://doi.org/10.1086/669042

[58] Treanor Mike. 2009. Newsgames - Procedural Rhetoric Meets Political Cartoons. In DiGRA- Proceedings of the 2009 DiGRA International Conference: Breaking New Ground: Innovation in Games, Play, Practice and Theory. Brunel University, London, UK, 9. http://www.digra.org/wp-content/uploads/digital-library/09300. 09505.pdf

[59] Peter Mitchell and Danielle Ropar. 2004. Visuo-spatial abilities in autism: A review. Infant and Child Development: An International fournal of Research and Practice 13, 3 (2004), 185-198. Publisher: Wiley Online Library.

[60] Tony Morelli, Lauren Lieberman, John Foley, and Eelke Folmer. 2014. An exergame to improve balance in children who are blind. In FDG. Society for the Advancement of the Science of Digital Games, Ft. Lauderdale, FL, 4. http: //www.fdg2014.org/proceedings.html

[61] Martez Mott, Edward Cutrell, Mar Gonzalez Franco, Christian Holz, Eyal Ofek, Richard Stoakley, and Meredith Ringel Morris. 2019. Accessible by Design: An Opportunity for Virtual Reality. In 2019 IEEE International Symposium on Mixed and Augmented Reality Adjunct (ISMAR-Adjunct). IEEE, Beijing, China, 451-454. https://doi.org/10.1109/ISMAR-Adjunct.2019.00122

[62] Michael J. Muller. 2003. Participatory design: the third space in HCI. Humancomputer interaction: Development process 4235 (2003), 165-185.

[63] Elena Márquez Segura, Laia Turmo Vidal, and Asreen Rostami. 2016. Bodystorming for movement-based interaction design. Human Technology 12, 2 (Nov. 2016), 193-251. https://doi.org/10.17011/ht/urn.201611174655

[64] Phil Parette and Marcia Scherer. 2004. Assistive technology use and stigma. Education and Training in Developmental Disabilities 39, 3 (2004), 217-226. Publisher: JSTOR.

[65] Heidi Parisod, Anni Pakarinen, Anna Axelin, Riitta Danielsson-Ojala, Jouni Smed, and Sanna Salanterä. 2017. Designing a Health-Game Intervention Supporting Health Literacy and a Tobacco-Free Life in Early Adolescence. Games for Health Journal 6, 4 (Aug. 2017), 187-199. https://doi.org/10.1089/g4h.2016.0107

[66] Brian A Primack, Mary V Carroll, Megan McNamara, Mary Lou Klem, Brandy King, Michael Rich, Chun W Chan, and Smita Nayak. 2012. Role of video games in improving health-related outcomes: a systematic review. American journal of preventive medicine 42, 6 (2012), 630-638. 
[67] Halley P. Profita, Abigale Stangl, Laura Matuszewska, Sigrunn Sky, Raja Kushalnagar, and Shaun K. Kane. 2018. "Wear It Loud" How and Why Hearing Aid and Cochlear Implant Users Customize Their Devices. ACM Transactions on Accessible Computing (TACCESS) 11, 3 (2018), 1-32. Publisher: ACM New York, NY, USA.

[68] Josephine M Randel, Barbara A Morris, C Douglas Wetzel, and Betty V Whitehill 1992. The effectiveness of games for educational purposes: A review of recent research. Simulation \& gaming 23, 3 (1992), 261-276. Publisher: Sage Publications Sage CA: Thousand Oaks, CA.

[69] Tom Salinsky and Deborah Frances-White. 2008. The improv handbook: the ultimate guide to improvising in comedy, theater, and beyond. Continuum, New York. OCLC: ocn225846984.

[70] Pat L. Sample. 1996. Beginnings: Participatory action research and adults with developmental disabilities. Disability \& Society 11, 3 (1996), 317-332.

[71] Charles E Schaefer. 2003. Play Therapy with Adults. Wiley, Hoboken, New Jersey. https://nbn-resolving.org/urn:nbn:de:101:1-2014112511845 OCLC: 897594749.

[72] Richard Schechner. 1988. Playing. Play \& Culture 1, 1 (1988), 3-19. Place: US Publisher: Human Kinetics Publishers, Inc.

[73] John Sharp and David Thomas. 2019. Fun, taste \& games: an aesthetics of the idle, unproductive, and otherwise playful. The MIT Press, Cambridge, MA.

[74] Kristen Shinohara and Jacob O. Wobbrock. 2011. In the shadow of misperception: assistive technology use and social interactions. In Proceedings of the 2011 annual conference on Human factors in computing systems - CHI '11. ACM Press, Vancouver, BC, Canada, 705. https://doi.org/10.1145/1978942.1979044

[75] Miguel Sicart. 2014. Play matters. MIT Press, Cambridge, MA, USA. http://www. vlebooks.com/vleweb/product/openreader?id=none\&isbn=9780262325967 OCLC 1124406508.

[76] Roger O. Smith, Marcia J. Scherer, Rory Cooper, Diane Bell, David A. Hobbs, Cecilia Pettersson, Nicky Seymour, Johan Borg, Michelle J. Johnson, Joseph P. Lane, S. Sujatha, P. V. M. Rao, Qussai M. Obiedat, Malcolm MacLachlan, and Stephen Bauer. 2018. Assistive technology products: a position paper from the first global research, innovation, and education on assistive technology (GREAT) summit. Disability and Rehabilitation: Assistive Technology 13, 5 (July 2018), 473-485. https://doi.org/10.1080/17483107.2018.1473895 Publisher: Taylor \& Francis_eprint: https://doi.org/10.1080/17483107.2018.1473895.

[77] Hayeon Song, Wei Peng, and Kwan Min Lee. 2011. Promoting exercise selfefficacy with an exergame. fournal of health communication 16, 2 (2011), 148-162. Publisher: Taylor \& Francis.

[78] Henrik Sproedt. 2012. Play. learn. innovate. Books On Demand, McFarland, WI OCLC: 939494986
[79] Jaakko Stenros. 2014. Behind games: Playful mindsets and transformative practices. In The Gameful World: Approaches, Issues, Applications, MIT Press, Cambridge. MIT Press, Cambridge, MA, USA, 201-222.

[80] Molly Follette Story. 1998. Maximizing Usability: The Principles of Universal Design. Assistive Technology 10, 1 (June 1998), 4-12. https://doi.org/10.1080/ 10400435.1998.10131955

[81] Kenneth T Strongman and Christopher DB Burt. 2000. Taking breaks from work: An exploratory inquiry. The fournal of psychology 134, 3 (2000), 229-242.

[82] Tiffany Thang. 2018. PhD Forum: Strengthening Social Emotional Skills for Individuals with Developmental Disabilities Through Virtual Reality Games. In 2018 IEEE International Conference on Smart Computing (SMARTCOMP). IEEE, Taormina, 242-243. https://doi.org/10.1109/SMARTCOMP.2018.00061

[83] Nicole Turygin, Johnny L. Matson, and Hilary Adams. 2014. Prevalence of cooccurring disorders in a sample of adults with mild and moderate intellectual disabilities who reside in a residential treatment setting. Research in Developmental Disabilities 35, 7 (July 2014), 1802-1808. https://doi.org/10.1016/j.ridd.2014.01.027

[84] Jelle van Dijk, Niels Hendriks, Christopher Frauenberger, Fenne Verhoeven, Karin Slegers, Eva Brandt, and Rita Maldonado Branco. 2016. Empowering people with impairments: how participatory methods can inform the design of empowering artifacts. In Proceedings of the 14th Participatory Design Conference: Short Papers, Interactive Exhibitions, Workshops - Volume 2. ACM, Aarhus Denmark, 121-122. https://doi.org/10.1145/2948076.2948101

[85] Karen Ward and Jordan S. Trigler. 2001. Reflections on Participatory Action Research With People Who Have Developmental Disabilities. Mental Retardation 39, 1 (Feb. 2001), 57-59. https://doi.org/10.1352/0047-6765(2001)039<0057: ROPARW>2.0.CO;2 Publisher: Allen Press.

[86] Voravika Wattanasoontorn, Imma Boada, Rubén García, and Mateu Sbert. 2013. Serious games for health. Entertainment Computing 4, 4 (2013), 231-247.

[87] PJ White, Hannah R Marston, Linda Shore, and Robert Turner. 2020. Learning from COVID-19: Design, Age-friendly Technology, Hacking and Mental Models. Emerald Open Research 2, 21 (2020), 21. Publisher: Emerald Publishing Ltd.

[88] Chauncey Wilson. 2013. Brainstorming and beyond a user-centered design method. Elsevier Science, Edinburgh, London. https://learning.oreilly.com/library/view//9780124071575/?ar OCLC: 1145028315

[89] Jacob O. Wobbrock, Shaun K. Kane, Krzysztof Z. Gajos, Susumu Harada, and Jon Froehlich. 2011. Ability-Based Design: Concept, Principles and Examples. ACM Transactions on Accessible Computing 3, 3 (April 2011), 1-27. https://doi.org/10. $1145 / 1952383.1952384$

[90] Eric Zimmerman. 2004. Narrative, interactivity, play, and games: Four naughty concepts in need of discipline. In First person: New media as story, performance, and game. MIT Press, Cambridge, MA, USA, 154-164. 